\title{
Estresse e Fatores Psicossociais
}

\author{
Stress and PsychosocialFactors \\ Stress y Factores Psychosociales
}
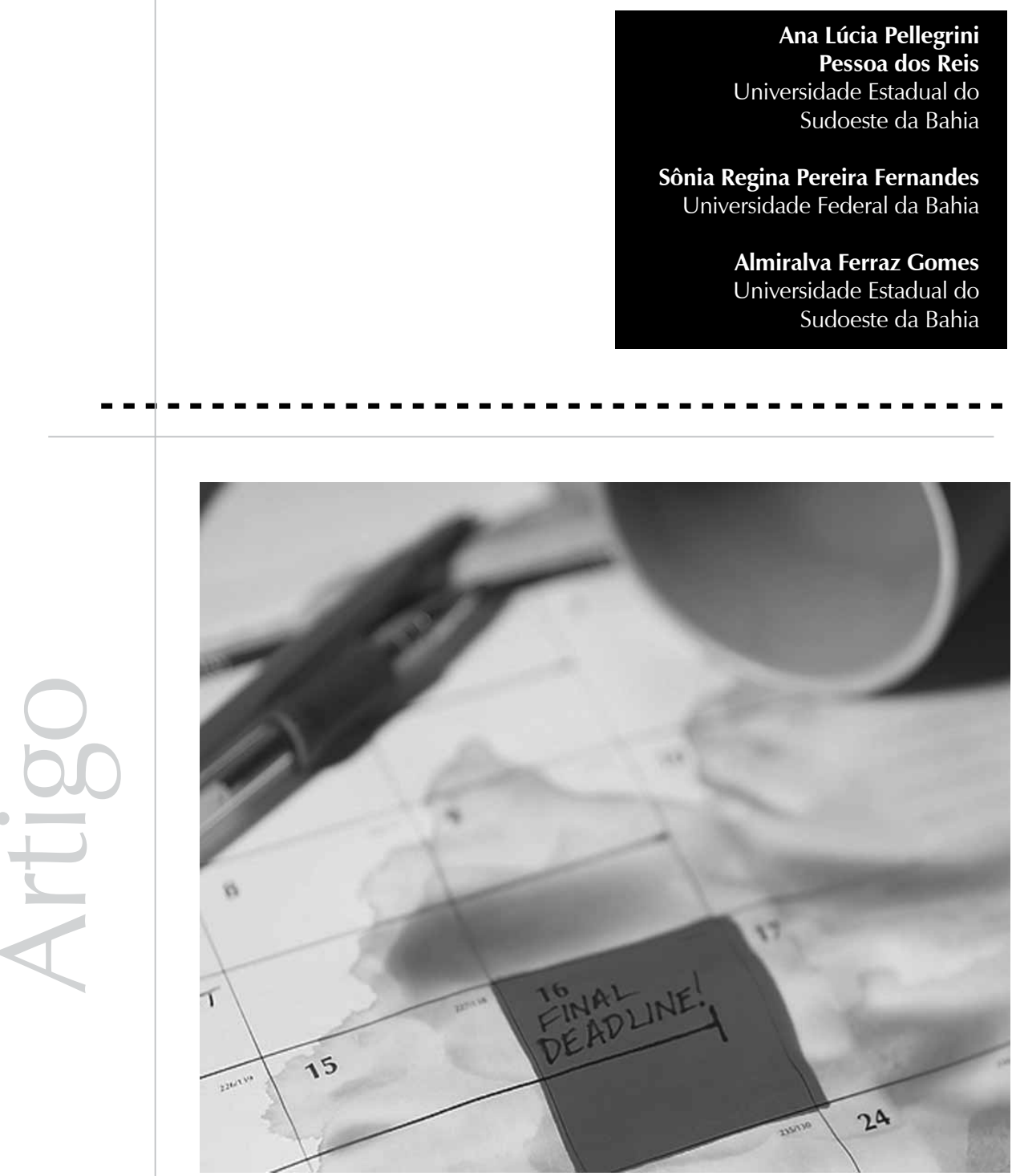
Resumo: $O$ interesse pelo estudo do estresse no trabalho tem sido crescente na literatura científica, particularmente nos últimos anos. Uma razão para o aumento de pesquisas sobre esse tema está nas repercussões das condições e da organização do trabalho na saúde e no bem-estar dos empregados e, em consequência, no funcionamento e na efetividade das organizações. Na economia, o impacto negativo dessa variável tem sido estimado com base na suposição e nos achados de que trabalhadores estressados diminuem seu desempenho e aumentam os custos das organizações com problemas de saúde, com o aumento do absenteísmo, da rotatividade e do número de acidentes no local de trabalho. Tendo em vista a importância do tema, o presente trabalho se propõe a analisar os modelos teóricos sobre estresse ocupacional e fatores psicossociais. Neste artigo, portanto, apresentaremos os princípios dos principais modelos que discutem a relação entre os fatores psicossociais do trabalho e o estresse ocupacional: modelo demandacontrole, modelo demanda-controle-apoio social, modelo do desequilíbrio esforço-recompensa, modelo ecológico e a proposta do National Institute for Occupational and Health (NIOSH).

Palavras-Chave: Estresse. Fatores psicossociais. Saúde do trabalhador. Trabalho.

\begin{abstract}
The interest for the study of the stress in the labor world has been growing in the academic literature, particularly in the last years. A reason for the increase of research on this theme is the negative impact of the occupational stress in the health and in the satisfaction of employees and, consequently, in the operation and in the effectiveness of the organizations. In the economy, the negative impact of this variable has been estimated based on the supposition and on the evidence that stressed workers reduce their acting and increase the costs of the organizations with health problems, increase of absence, employee turnover and the number of accidents in the labor environment. The present work proposes to analyze the theoretical models about occupational stress and psychosocial factors. For this, we will present approaches that study the occupational stress and the psychosocial factors in the labor environment. In this article, therefore, we will present the principal models that discuss psychosocial factors and occupational stress: job-demand-control, job demand control support model, effort-reward imbalance model, ecological model and the National Institute for Occupational and Health (NIOSH) proposal.
\end{abstract}

Keywords: Stress. Psychosocial factors. Occupational health. Work.

Resumen: El interés para el estudio del stress en el trabajo ha estado creciendo en la literatura académica, particularmente en los últimos años. Una razón para el aumento de investigaciones sobrelas repercusiones de las condiciones y lorganizacion de trabajo para la salud y bienestar de los empleados y, por consiguiente, en el funcionamiento y en la efectividad de las organizaciones. En la economía, el impacto negativo de esta variable se ha estimado con la base en la suposición y en la evidencia de que trabajadores tensos reduzca su desenpeño y aumenten los costos a las organizaciones con los problemas de salud, con el aumento de lo absentismo, de la variación constante de los empleados y del número de accidentes en el ambiente laboral. El presente trabajo propone analizar los modelos teóricos sobre la stress ocupacional y el factores psychosociales. Para tanto, nosotros presentaremos los principios de los principales modelos que estudian la relación entre los factores psychosociales del trabajo y lo stress ocupacional: el Modelo Demanda-controle; el Modelo Demanda-Controle-Apoyo Social; el Modelo del Desequilibrio Esfuerzo-Recompensa; el Modelo Ecológico y la propuesta del National Institute for Occupational and Health (NIOSH).

Palavras clave: Stress. Factores psychosociales. Salud del trabajador. Trabajo.

As transformações econômicas, tecnológicas e institucionais que vêm ocorrendo, principalmente nas três últimas décadas, têm impactado profundamente a forma de gerir as organizações, inclusive no que diz respeito à gestão de pessoas. Tais mudanças têm gerado repercussões diferenciadas na saúde e na integridade do trabalhador.

Para sobreviver, as organizações desenvolvem um novo patamar organizacional coerente com o seu novo ambiente, que se caracteriza por ser cada vez menos previsível e por apresentar crescente instabilidade; além disso, alteram sua organização do trabalho para uma estrutura que privilegia a integração e a flexibilidade, incentivando o dinamismo, a participação do trabalhador, o trabalho cooperativo e em equipe, o incremento da velocidade, a polivalência e a rotação de tarefas. Tais práticas têm como filosofia a superação constante, e visam a melhorar e a modificar sempre a organização da força de trabalho para, permanentemente, promover a evolução tecnológica e obter competitividade.

Essa busca frenética por um lugar e uma posição melhor no mercado denuncia 
De acordo com Couto e Moraes (2000), o atual ambiente de negócios é caracterizado por profundas racionalizações, corte de pessoal e alta velocidade de mudanças tecnológicas. a existência de um descompasso entre a evolução dos modelos de gestão e as políticas de gestão em saúde e segurança. Pesquisadores escandinavos (Doef \& Maes, 1999; Karlquist, Hagberg, Koster, Wenemark, \& Nell, 2003; Marmot, 1999; Piko, 2003; Tsutsumi, Kayaba, Theorell, \& Siegrist, 2001) apresentam resultados de estudos sobre os impactos das novas tecnologias informacionais na saúde física e mental, indicando consequências como problemas musculoesqueléticos e visuais e doenças cardiovasculares e mentais relacionados ao trabalho. Os estudos sugerem que tanto fatores físicos quanto psicossociais devem respeitar as necessidades e as demandas física e mental do trabalhador. Os autores mencionam aspectos psicossociais relativos à investigação de várias causas de insatisfação, identificando fatores como baixo controle sobre o trabalho, tensão causada pelo ritmo acelerado, grande demanda quantitativa e qualitativa, impedimento de contatos e conflitos interpessoais, ausência de apoio social, baixa possibilidade de desenvolvimento, pressão ocasionada pelo controle automatizado, tarefas rotineiras e fragmentadas, monotonia e tédio.

De acordo com Couto e Moraes (2000), o atual ambiente de negócios é caracterizado por profundas racionalizações, corte de pessoal e alta velocidade de mudanças tecnológicas. Nesse ambiente, evidencia-se a existência de trabalhos de alta densidade, que envolvem o uso permanente de sistemas computadorizados. Esses trabalhos apresentam como características o uso constante da memória imediata, o grande número de microdecisões e também a possibilidade de existência de carga afetiva na tarefa, favorecendo, assim, a fadiga. São sintomas decorrentes desses trabalhos: esquecimentos, erros, incapacidade de solução de vários problemas simultaneamente e, em consequência, frustração para o indivíduo, com ansiedade e temor do fracasso.
Por conseguinte, a organização também sofre prejuízos decorrentes dessas falhas. Um aspecto do trabalho de alta densidade que chama a atenção é a autoaceleração. Esse mecanismo leva as pessoas a acelerar cada vez mais o seu ritmo, gerando uma grande dificuldade de reduzi-lo e chegando ao ponto de o indivíduo se negar, por exemplo, a tirar férias. Essa autoaceleração é especialmente importante no trabalho de gerentes e executivos, e pode gerar a síndrome obsessivo-compulsiva denominada workaholism.

Em geral, os novos modelos de gestão, além de incorporarem características da tecnologia informacional por alterarem as formas de controle do trabalho mediante maior espaço de participação dos trabalhadores na formulação das estratégias gerais das empresas, ampliam a co-responsabilidade com os resultados. Esses trabalhadores, que, geralmente, ficam em ambientes mais limpos, com eliminação das cargas de trabalho físicas e mecânicas, entre outras, e que, portanto, teoricamente teriam a possibilidade de gozar de melhor saúde, estão expostos a novas cargas de trabalho, tanto no aspecto cognitivo como no psicoemocional.

Dentro desse enfoque, a relação entre gestão do trabalho e saúde, ainda que em número insuficiente, tem sido estudada sob diversos ângulos, variando conforme as áreas de conhecimento e a natureza da problemática analisada, sendo a abordagem do estresse largamente utilizada para avaliar trabalho e saúde.

Deve-se ressaltar a importância da análise sobre estresse ocupacional e fatores psicossociais uma vez que esses elementos poderão subsidiar programas de intervenção nos ambientes de trabalho visando à promoção de saúde do trabalhador. 


\section{Estresse Ocupacional}

As estimativas da Organização Mundial da Saúde - OMS - ressaltam que os transtornos mentais menores acometem aproximadamente $30 \%$ dos trabalhadores ocupados, e os transtornos mentais graves, entre 5 e $10 \%$. No Brasil, dados do Instituto Nacional do Seguro Social - INSS - referentes à concessão de benefícios previdenciários de auxílio-doença, por incapacidade para o trabalho superiores a 15 dias e de aposentadoria por invalidez ou incapacidade definitiva para o trabalho, demonstram que os transtornos mentais ocupam o terceiro lugar entre as causas dessas ocorrências (Brasil, 2002).

Com base em dados como esses, o Ministério da Saúde (Brasil, 2002) sugeriu atenção para as condições de insegurança no emprego, subemprego e segmentação crescente do mercado de trabalho, pois entende que o surgimento de novas formas de adoecimento mal caracterizadas como o estresse, a fadiga física e mental e outras manifestações de sofrimento relacionadas ao trabalho tem forte relação com os processos internos de reestruturação da produção, com o enxugamento de quadro de funcionários e com a incorporação tecnológica. Para melhor entender esse fenômeno, o Ministério da Saúde tem incentivado a realização de pesquisas para que propostas coerentes e efetivas de intervenção possam ser traçadas.

Codo, Soratto e Vasques-Menezes (2004) enfatizam que, embora a imprecisão do termo estresse se estenda ao meio científico, as várias áreas que o adotam defendem a concepção de que o estresse é o resultado de um estado de desequilíbrio tanto da relação indivíduo-ambiente de trabalho quanto da relação demanda-recursos. Ainda que existam diversos modelos teóricos, verificase uma certa concordância na definição do estresse ocupacional. Segundo Codo et al. (2004), alguns modelos concordam que a relação entre estímulos externos e estresse pode ser moderada por características individuais e situacionais. Essa abordagem busca a aproximação dos possíveis efeitos do trabalho sobre o indivíduo trabalhador, possibilitando a avaliação de modos de intervenção mesmo em situações em que as fontes de estresse não possam ser eliminadas.

É interessante salientar que o estudo do estresse tem sido dominado pela perspectiva clínica, como um fenômeno psicofisiológico decorrente da percepção individual de desajustes entre as demandas do ambiente e a capacidade de respostas do indivíduo. Nessa perspectiva, o estresse tem consequências fisiológicas, psicológicas e comportamentais que são mediadas pela percepção, com foco na susceptibilidade do indivíduo, cujas intervenções são dirigidas para o desenvolvimento de estratégias individuais de enfrentamento. Por outro lado, estudos sobre a saúde do trabalhador enfatizam a prevenção primária no local de trabalho por meio de identificação e de modificação de condições laborais que possam constituir perigo para a saúde. De acordo com essa visão, a chave da questão do estresse no trabalho é reconhecer a dinâmica da sua etiologia (Kalimo, 1988; National Institute for Occupational and Health [NIOSH], 2004).

Ao se considerar que o estresse tem etiologia múltipla, têm-se formulado e validado diferentes modelos para explicar suas causas e, na atualidade, tem crescido a preocupação com os fatores psicossociais no trabalho. Vários estudos enfatizam que constantes mudanças ocorridas nos locais de trabalho provocam mudanças no meio psicossocial, funcionando como precipitador de doenças. Independentemente do modo de vida e da vulnerabilidade dos trabalhadores, fatores 
psicossociais associados a novos métodos de trabalho têm-se apresentado como uma das causas principais do comprometimento da saúde da população trabalhadora (Benach et al., 2002; Borg \& Kristensen, 1999; Fernandes, 1999; Figueiras \& Hippert, 2002; Kalimo, 1988; Lima, 2002; Marmot, 1999; SeligmanSilva, 1997). Nesse sentido, os estudos sobre estresse ocupacional, geralmente, têm incorporado fatores psicossociais como elementos presentes nesse processo.

\section{Fatores Psicossociais No Trabalho}

Há consenso entre alguns autores (ElBatawi, 1988; Levi, 1988; Kalimo, 1988; Fernandes, 1999; Villalobos, 2004) quanto à concepção de os fatores psicossociais no trabalho constituírem elementos do ambiente organizacional que, ao serem experienciados pelos trabalhadores, sofrem influências de variáveis de caráter individual. Ainda que haja concordância entre os autores, ao abordálos, tratam diferentemente de tais fatores, situando-os por vezes dentro de um macro contexto histórico e social ou enfatizando suas origens e efeitos.

Ao estudar a relação entre estresse e trabalho, Villalobos (2004) considera que os fatores psicossociais do trabalho representam um conjunto de percepções e experiências, ou seja, consistem em interações entre o trabalho, o ambiente laboral, as condições da organização e as características pessoais do trabalhador, suas necessidades, cultura, experiências, estilo de vida e sua percepção de mundo. Inclui, entre os principais fatores psicossociais do trabalho geradores de estresse, aspectos da organização, gestão e processo de trabalho e as relações humanas. O autor situa a organização em um contexto histórico, com o seu conjunto de problemas demográficos, econômicos e sociais, no qual interage; contempla, portanto, as variáveis do macrocontexto, sua interação com os elementos do ambiente organizacional, e o indivíduo, com sua multideterminação.

Já Fernandes, ao avaliar os fatores psicossociais do trabalho e sua relação com a saúde psíquica, evidencia também a influência de aspectos intrínsecos ao indivíduo na produção do estresse, considerando que, nessa relação, intervêm as atitudes, o estilo de vida, as estratégias de cooping e apoio social assim como a vulnerabilidade e a resistência ao estresse. Embora não desconsidere os fatores externos à organização, enfatiza mediadores da situação ou do indivíduo que podem fortalecer ou debilitar a influência de fatores do ambiente de trabalho.

Kalimo, assim como Fernandes, ao discorrer sobre os fatores psicossociais e suas implicações na saúde do trabalhador, enfatiza a interação de aspectos do ambiente de trabalho e de variáveis de caráter individual e subjetivo, como as experiências passadas, os fatores genéticos e as condições de vida, que vão influenciar o modo como os indivíduos experimentam e interpretam suas vivências. Embora também considere as diferenças individuais como fatores que intervêm na relação trabalho-saúde, ressalta a importância de avaliar tais aspectos com enfoque no grupo, já que julga que, quando a influência de um fator psicossocial é intensa, é menor a importância da vulnerabilidade individual. Levi (1988) identifica a origem dos estímulos psicossociais em processos sociais que se situam em uma estrutura social e afetam o organismo por meio da sua percepção e experiência. Considera, em seu modelo teórico, no que se refere aos aspectos psicossociais, a influência de variáveis interatuantes extrínsecas e intrínsecas, sociais, mentais ou físicas que têm como função modificar a ação dos fatores causais na fase do mecanismo, do precursor ou da enfermidade, promovendo ou evitando o 
processo que culmina com a doença. Esse autor amplia as concepções anteriores na medida em que situa os fatores psicossociais em um enfoque sistêmico, portanto, dotado de retroalimentação contínua.

Embora a abordagem dos fatores psicossociais no trabalho tenha sido o tema do seu estudo como precipitador do estresse, El-Batawi classifica esses fatores em duas categorias: os que têm efeitos negativos na saúde e os que podem contribuir positivamente para o bemestar dos trabalhadores. Esse autor enfatiza o trabalho como fator de fomento à saúde, não se devendo, portanto, subestimar os seus aspectos positivos e efeitos estruturadores.

Ainda que não façam referência direta a fatores psicossociais, outros autores, ao abordarem os impactos dos elementos do ambiente organizacional na saúde mental do trabalhador (Figueiras \& Hippert, 2002; Lima, 2002; Monteiro \& Gomes, 1998; Seligman-Silva, 1997), discutem a sua interação com variáveis individuais, conquanto os estudos desenvolvidos utilizam abordagens diferenciadas, que variam entre a quantitativa, a qualitativa e a mista.

\section{Fatores Psicossociais e Estresse Ocupacional}

Neste artigo, apresentaremos os princípios dos modelos mais conhecidos que discutem fatores psicossociais e estresse ocupacional. O modelo demanda controle e o modelo do desequilíbrio esforço-recompensa são os modelos mais adotados em se tratando de fatores psicossociais do estresse no trabalho (Marmot, 1999; Tsutsumi et al., 2001). Esses modelos têm sido predominantes, possivelmente, em função das evidências empíricas e, principalmente, por contemplarem fatores do ambiente psicossocial de trabalho nas organizações em contextos globalizados e que valorizam as novas tecnologias. Levi (1998) considera que esses modelos são complementares ao modelo ecológico, apresentado por Kagan e Levi, em 1978, que também será discutido aqui. Não obstante a importância desses modelos, a despeito dos desafios metodológicos a serem transpostos no campo de estudo do estresse e trabalho, incluiremos a proposta do $\mathrm{NIOSH}$, por se tratar de uma abordagem integradora que enfatiza o enfoque coletivo para compreensão do estresse no ambiente de trabalho. Ressaltamse, também, como determinantes principais, os fatores do ambiente, sem ignorar variáveis moderadoras e modificadoras de efeito que possam influenciar a relação trabalho e saúde.

\section{O modelo demanda-controle e o modelo demanda-controle-apoio social}

O modelo demanda controle (job-demandcontrol - JDC ou job strain model), proposto por Karasek (1979), considera que o strain resulta da interação das demandas de trabalho e da latitude de decisão no trabalho. A latitude de decisão é definida como o controle do trabalho pelo indivíduo sobre suas tarefas e sua conduta durante o dia de trabalho. Duas medidas compõem esse controle: autoridade para decidir e uso de habilidades, que representam medidas agregadas de aspectos diferentes do controle no trabalho - habilidade para a realização de tarefas, organização da tarefa (aprendizagem de coisas novas, criatividade, iniciativa, realização de tarefas repetidas ou variadas, uso e desenvolvimento de habilidades especiais), influência na política organizacional, controle sobre incertezas potenciais e recursos para decisão. O uso de habilidades intelectuais representa uma oportunidade para realizar julgamento. Como 
enfatiza o autor, isso fortalece o sentimento de eficácia e a habilidade para enfrentar o ambiente, e funciona como fator protetor do estresse. No entanto, constrangimentos na tomada de decisão representam problemas para o indivíduo que trabalha com poucas oportunidades para tomar decisões em face da pressão de produção, o que constitui fonte de tensão no trabalho.

Quanto às demandas de trabalho, referemse a estressores psicológicos envolvidos na realização da carga de trabalho, tais como: tarefas inesperadas, volume de trabalho, nível de atenção e concentração requerida, pressão de tempo, interrupção das tarefas, dependência da realização de tarefas por outros e conflito de papel. Por sua vez, um conjunto de indicadores avalia os sintomas de tensão agrupados em dois fatores: exaustão e depressão. O indicador de exaustão constitui resposta de cansaço pela manhã e completa exaustão à noite. Já o indicador de depressão consiste de aspectos como nervosismo, ansiedade, problemas com o sono, preocupação e depressão, com previsão na variação nos níveis de tensão mental.

Conforme Karasek (1979), os estudos têm registrado associação entre medidas de satisfação e depressão, indicando relação com o nível de atividade do trabalho. Assim, trabalhos mais ativos (alta demanda e alto controle) estão associados a satisfação e depressão reduzida, mesmo que sejam mais demandadores. Ainda para o autor, uma implicação importante desses estudos é que pode ser possível melhorar a saúde mental no trabalho sem sacrificar a produtividade, aumentando o controle do trabalho independentemente das mudanças nas demandas. Além disso, mudanças na estrutura administrativa são importantes para melhorar a habilidade do trabalhador na tomada de decisão e para aumentar sua influência em decisões organizacionais, permitindo o uso de seu potencial. Além dos trabalhos caracterizados como de alta exigência e trabalhos ativos, o autor também distingue duas outras experiências decorrentes ainda da combinação entre controle do trabalho e demandas: os trabalhos passivos, aqueles com baixa demanda e baixo controle, e os de baixa exigência, assim denominados em função da combinação de baixas exigências e alto controle. Karasek prevê também que os trabalhos de alta exigência são geradores de riscos psicológicos e de adoecimento psíquico, com indicação de reações adversas como fadiga, ansiedade, depressão e doenças físicas (Karasek \& Theorell, 1990). O autor sugere também que há motivação para desenvolver novos comportamentos no trabalho ativo, ao contrário do trabalho passivo, que pode levar à redução da atividade do indivíduo e da sua capacidade de produzir soluções para as atividades e os problemas enfrentados.

Tal proposta tem sofrido críticas, ainda que agregue contribuições para melhoria da vivência do indivíduo no trabalho e seja largamente utilizada. Embora demonstre o impacto das demandas e o controle do trabalho no estresse, o JDC não identifica estressores específicos do ambiente de trabalho dentro dessas categorias, que poderiam ser modificados para reduzir o estresse. Tanto as demandas de trabalho como o controle do trabalho são compostos de muitos estressores potenciais. Esse modelo delineia a interação entre estes grupos de estressores, mas não indica os que são críticos dentro de cada grupo. Embora o JDC enfatize a interação de características do trabalho que são responsáveis pelo desenvolvimento do estresse, também não focaliza especificamente a interação entre o indivíduo e o ambiente nem avalia características individuais e valores. Também há inconsistências na aplicação do modelo, atribuídas ao uso inadequado dos conceitos 
de controle do trabalho e demandas de trabalho e à realização de estudos com análises de nível ocupacional, com potencial confusão com variáveis socioeconômicas. Sugere-se, a partir dos resultados obtidos com o modelo de Karasek (1979), a ampliação do foco para além de duas características do trabalho, como a avaliação de suportes interpessoais e outros aspectos do trabalho. Críticos salientam que os fatores que influenciam na tensão no trabalho não provêm apenas do ambiente laboral. Esses aspectos interagem com características individuais que constituem fatores relevantes (Bliese \& Castro, 2000; Rodríguez, Bravo, Pieró, \& Eschoufeli, 2001).

Ao modelo teórico proposto por Karasek (1979), baseado em uma dupla matriz de demanda e controle, foi incorporado posteriormente o fator apoio social (Karasek \& Theorell, 1990). O papel do apoio social no job demand control support model (JDCS) foi teoricamente desenvolvido por Jeffrey V. Johnson, em 1986 (Theorell, 1998), para quem o apoio social de superiores e colegas de trabalho pode funcionar como amortecedor, buffer, na relação entre demandas e controle do trabalho. O apoio social caracteriza o clima social do ambiente de trabalho e tem como componente tanto a relação emocional que o trabalho comporta quanto o suporte instrumental.

Martinez (2004) enfatiza que tanto o apoio social quanto o controle do trabalho são fatores que têm sofrido influência das mudanças na organização do trabalho, e as intervenções preventivas que buscam modificar os riscos psicossociais, em geral, afetam essas duas dimensões.

Testes do modelo JDCS encontram evidências para sugerir a inclusão do apoio social como importante extensão do JDC, com dados de que, na relação interativa entre controle do trabalho e demandas de trabalho, o apoio social tem papel moderador no controle do impacto negativo da combinação dessas variáveis (Bliese \& Castro, 2000; Doef \& Maes, 1999).

\section{O modelo do desequilíbrio esforço- recompensa}

A suposição núcleo do modelo do desequilíbrio esforço-recompensa (effortreward imbalance model-ERI), desenvolvido por Siegrist (1998), sustenta que a função do trabalho na vida adulta é crucial entre as funções autorregulatórias como a autoestima, a autoeficácia e a estrutura social de oportunidades. Nessa perspectiva, a disponibilidade de status ocupacional está associada com opções recorrentes de contribuir e atuar, de ser recompensado ou estimado e de pertencer a um grupo. O esforço no trabalho é despendido como parte de um processo de troca, organizado socialmente, cujas recompensas sociais constituem três dimensões de gratificação ocupacional - dinheiro, estima e status - que, nessa abordagem, se referem às condições de controle. O modelo do desequilíbrio esforçorecompensa defende o ponto de vista de que a falta de reciprocidade entre custos e ganhos, como condições de alto custo e baixo ganho, gera um estado de aflição que pode levar a reações associadas de tensão com efeitos de longo prazo adversos para a saúde física e mental. Quanto ao controle de status, esse modelo considera que ameaças são prováveis de ocorrer quando é interrompida ou perdida a continuidade das funções ou dos papéis sociais. Sob essas circunstâncias, o controle sobre recompensas interpessoais básicas é restrito e, como consequência, a autoestima e o bem-estar emocional são prejudicados.

O modelo apresenta como exemplo situações de desligamento ou instabilidade 
no trabalho, como também condições de baixa recompensa e baixa segurança na vida ocupacional verificadas em situações de mudança ocupacional forçada, mobilidade para baixo, falta de perspectiva de promoção ou trabalhos mantidos com condição inconsistente de status. Portanto, ter um emprego demandador, mas instável, que não oferece perspectiva de promoção, é exemplo de contexto de trabalho estressante. Siegrist chama a atenção para o mercado de trabalho na economia globalizada, que se caracteriza pelo baixo controle de status como reflexo crescente de carreiras de trabalho fragmentadas, de instabilidade no trabalho, de redundância e de mobilidade ocupacional forçada (Siegrist, 1998). Assim, o autor define duas fontes de alto esforço: a fonte extrínseca, demandas no trabalho, e uma fonte intrínseca, motivações dos indivíduos trabalhadores. Quanto à motivação dos indivíduos, ele considera que a necessidade de controle funciona como uma estratégia de coping para lidar com as demandas de trabalho.

Folkman e Lazarus (1980) conceituam coping como um conjunto de esforços cognitivos e comportamentais utilizados pelos indivíduos como estratégia para lidar com demandas internas ou externas em situação de estresse, avaliadas como sobrecarga ou excesso aos recursos pessoais. Constituem ações deliberadas que podem ser aprendidas, usadas e descartadas, e funcionam como mediador entre um estressor e o resultado dele advindo.

Folkman e Lazarus ainda propõem duas categorias de coping: uma que é focalizada no problema, e outra, na emoção. O coping focalizado na emoção é definido como um esforço para regular o estado emocional que é associado ao estresse ou que é o resultado de eventos estressantes, tem como função alterar o estado emocional do indivíduo e é dirigido a um nível somático ou psíquico para reduzir a sensação física desagradável de um estado de estresse. Já o coping focalizado no problema constitui um esforço para atuar na situação que originou o estresse, buscando alterá-la e, assim, diminuir a tensão. O coping focalizado no problema pode ser direcionado externamente, e inclui estratégias tais como negociar para resolver um conflito ou solicitar ajuda de outra pessoa, ou internamente, que, em geral, compreende reestruturação cognitiva como, por exemplo, a redefinição de um elemento estressor. A escolha da estratégia pelo indivíduo depende da avaliação da situação estressora, em que são julgados o risco envolvido e as opções para lidar com o problema. Se as situações forem avaliadas como modificáveis, o coping focalizado no problema tende a ser mais utilizado, enquanto aquele focalizado na emoção tende a ser empregado nas situações consideradas inalteráveis. Para os autores, qualquer tentativa para lidar com o estressor é vista como estratégia de coping, independentemente de seu resultado.

Com base na teoria de Folkman e Lazarus (1980) e Siegrist (1998) prevê ajustes cognitivos e comportamentais, estratégias de coping para lidar com uma condição de alto custo e baixo ganho como consequência dos processos de avaliação cognitiva; evidencia, entretanto, que uma limitação dessa abordagem é a submissão dos processos de informação afetiva à consciência. Esse autor considera que o efeito negativo associado à experiência de desequilíbrio entre esforço e recompensa no trabalho não necessariamente está submetido ao despertar da consciência, especialmente em experiências diárias cronicamente recorrentes.

O modelo ERI difere do JDC em dois aspectos. O primeiro enfatiza mais a recompensa que o controle, e inclui aspectos do mercado de trabalho (salário, perspectiva de promoção, 
segurança no trabalho), aspectos do local de trabalho e também um componente intrínseco pessoal. O modelo ERI prevê também um estilo pessoal de coping para lidar com as demandas, denominado overcommitment, que define como um conjunto de atitudes, comportamentos e emoções que refletem um esforço excessivo em combinação com um forte desejo de ser reconhecido e estimado. Pessoas caracterizadas por overcommitent exageram seus esforços além dos níveis considerados apropriados. Adicionalmente, o modelo ERI incorpora duas fontes de informação, a situacional (extrínseca) e a pessoal (intrínseca) para avaliar experiências de estresse no trabalho (Tsutsumi et al., 2001).

\section{O modelo ecológico}

A ideia central do modelo proposto por Levi e Kagan (Levi, 1998), denominado modelo ecológico (ecological model), é de que as situações ocupacionais ocorrem em uma estrutura e em um processo social, enquanto os estímulos psicossociais atuam sobre o trabalhador, que se caracteriza por seu programa psicobiológico, ou seja, pela sua propensão a reagir segundo certos padrões que podem ser modificados, e interagir com variáveis como o apoio social e o repertório de coping, que podem funcionar como protetores.

Para Levi (1988), as reações são provocadas pela ação recíproca ou por desajustes entre as oportunidades e as exigências inerentes ao meio e às necessidades e expectativas individuais. Quando o ajuste não é bom, quando não se atende às necessidades ou quando há esforço em excesso, o organismo reage através de diversos mecanismos patógenos, que podem ser cognitivos, afetivos, comportamentais ou fisiológicos, e em certas condições de intensidade, frequência ou duração. Nessa perspectiva, torna-se importante, então, identificar os componentes desse sistema, assim como sua interação dinâmica, que determinam, aceleram o curso ou disparam o sintoma, para intervir preventiva ou terapeuticamente.

Levi (1988) considera ainda que esse processo constitui um sistema cibernético com contínuo feedback, e que é preciso conhecer o conteúdo de cada um desses aspectos, suas interações e a dinâmica de todo o sistema para que ações sejam implementadas. O autor também leva em conta o fato de os modelos de Karasek e Theorel - JDCS - e o modelo proposto por Siegrist - ERI - serem complementares ao modelo ecológico e suplementares entre si.

\section{O modelo do $\mathrm{NIOSH}$}

$\mathrm{O} \mathrm{NIOSH}$ define estresse no trabalho como as nocivas reações físicas e emocionais que ocorrem quando as exigências não se igualam à capacidade, aos recursos ou às necessidades do trabalhador, como resultado da sua interação com as condições de trabalho, o que pode levá-lo a doenças. Apoia a ideia de que as condições laborais têm papel principal nas causas do estresse, ainda que não se possa ignorar a importância das diferenças individuais e de outras situações que podem intervir para fortalecer ou debilitar a influência desses fatores (NIOSH, 2004).

O NIOSH (2004) distingue um conjunto de condições que pode causar o estresse ocupacional: o desenho do trabalho (trabalho pesado, descansos infrequentes, turnos e horas de trabalho longos, trabalhos frenéticos e de rotina, que têm pouco significado ou que não usam as habilidades dos trabalhadores que provêm pouco sentido de controle), o estilo da gestão (ausência de participação na tomada de decisão, falta de comunicação na organização, ausência de uma política 
favorável à vida da família), relações interpessoais (ambientes sociais inadequados, falta de apoio e ajuda de companheiras e supervisores), papéis no trabalho (expectativas de trabalho mal definidas ou impossíveis de alcançar, demasiada responsabilidade e funções múltiplas), preocupações com a carreira (insegurança no trabalho e falta de oportunidade para o crescimento pessoal, o fomento ou a ascensão, mudanças rápidas para as quais os trabalhadores não estão preparados) e condições ambientais (condições desagradáveis e perigosas).

Embora essa proposta do NIOSH (2004) não constitua um modelo consensual, não especifique o efeito da interação entre as várias condições de trabalho, nem explicite minuciosamente as capacidades, os recursos ou as necessidades dos trabalhadores, apresenta um modelo genérico que integra as abordagens anteriores e enfatiza as condições laborais como importantes elementos a serem priorizados em uma abordagem coletiva. A proposta considera também as variáveis mediadoras do indivíduo ou da situação, como as estratégias de coping e o apoio social, que intervêm no processo entre as condições estressoras de trabalho e o risco de doenças, também concebendo o estresse ocupacional como decorrente de fatores psicossociais presentes no ambiente de trabalho, que evidenciam desajustes entre as características das pessoas e o seu ambiente laboral.

\section{Considerações Finais}

O trabalho funciona como importante medida de integração social tanto pela dimensão de subsistência quanto pela dimensão simbólica, essencial na constituição das pessoas. Também ocupa um espaço central na dinâmica do seu investimento afetivo e, em consequência, condições de trabalho que favorecem a utilização das habilidades do trabalhador bem como possibilitam o controle do trabalho e oferecem apoio social podem proporcionar saúde e bem-estar. Por outro lado, situações de fracasso, mudanças de posição na hierarquia, impossibilidade de comunicação espontânea e manifestação de descontentamento relativa à organização ou ao trabalho, fatores relacionados ao tempo e ao ritmo, níveis de atenção e concentração para a realização de tarefas, combinados com o nível de pressão exercida, podem provocar tensão e, por conseguinte, sofrimento e distúrbios mentais. Esse sofrimento é evidenciado não apenas pelas doenças mas também pelos indicadores de absenteísmo e pelos conflitos interpessoais e extratrabalho (Brasil, 2002).

As abordagens aqui apresentadas constituem importantes modelos explicativos do estresse ocupacional e enfatizam, em uma perspectiva de saúde coletiva, que a sua prevenção deve focar a adaptação do trabalho às expectativas, habilidades e necessidades do trabalhador, através do monitoramento dos fatores psicossociais no trabalho. Verificamos que os modelos sequencialmente apresentados se complementam, incorporando gradativamente elementos que interagem, que vão desde aspectos do contexto de trabalho às características do indivíduo ou da situação, e que podem funcionar como moderadores ou determinantes do estresse ocupacional.

Apesar da evolução desses modelos de compreensão do estresse ocupacional, no sentido de concebê-los como multideterminados, ainda não se incorporam fatores externos ao ambiente de trabalho que podem influenciar na sua constituição. 
Contrariamente ao que poderíamos esperar, alguns autores (Aguiar, 2002; Antunes, 2002) registram que as novas técnicas de gestão empresarial não se diferenciam, na essência, das práticas antigas. Ao que parece, essas práticas aumentam o controle sobre os indivíduos pela adesão e pela interiorização de regras. Mesmo as políticas que convidam à participação e à autonomia têm os mesmos objetivos que as antigas práticas disciplinares, entretanto, possuem uma aparência mais sofisticada, lógica e científica quanto à gestão de pessoas. Os procedimentos são disciplinadores por ampliarem o controle sobre o indivíduo, e têm impacto na ordem política, econômica, ideológica e psicológica.

As práticas de gestão de pessoas implementadas na nossa realidade, que incorporam estratégias de participação dos trabalhadores, têm sido bastante questionadas, visto que essa participação tem sido visualizada quase sempre na perspectiva da adesão aos modelos organizacionais. Nesse sentido, é necessário que as organizações desenvolvam práticas de gestão que adotem a efetiva participação dos trabalhadores nos processos de trabalho de forma a possibilitar o bem-estar e a promoção da saúde psíquica. 
Ana Lúcia Pellegrini Pessoa dos Reis

Doutoranda em Saúde Coletiva pela Universidade Federal da Bahia, Bahia, BA - Brasil. E-mail: pellegrinireis@yahoo.com.br

Sônia Regina Pereira Fernandes

Doutora em Saúde Coletiva pela Universidade Federal da Bahia, Bahia, BA - Brasil.

E-mail: sonregina@gmail.com

\section{Almiralva Ferraz Gomes}

Doutora em Administração pela Universidade Federal de Lavras, Bahia, BA - Brasil.

E-mail: almiralva@gmail.com; almiralvag@yahoo.com

*Endereço para envio de correspondência:

Av. Paulo VI, 2240, AP 604 Itaigara, Salvador - Bahia - Brasil CEP 41810-001.

Recebido 5/2/2009, 1aㅡ Reformulação 29/3/2010, 2ª Reformulação 20/5/2010, Aprovado 24/5/2010. 


\section{Referências}

Aguiar, M. A. F. (2002). Psicologia aplicada à administração: globalização, pensamento complexo, teoria crítica e a questão ética nas organizações. São Paulo: Excellus.

Antunes, R. (2002). Os sentidos do trabalho: ensaios sobre a afirmação e a negação do trabalho. São Paulo: Boitempo.

Benach, J., Muntaner, C., Benavides, F. G., Amable, M., \& Jadar, P. (2002). A new occupational health agenda for a new work environment.Scandinavian Journal of Work, Environment \& Health, 28(3), 191-196.

Bliese, P. D., \& Castro, C. A. (2000). Rode clarity, work overload and organizational support: Multilevel evidence of the importance of support. Work \& Stress, 14(1), 65-73.

Borg, V. H., \& Kristensen, T. S. (1999). Psychosocial work environment and mental heath among travelling salespeople. Work \& Stress, 13(2), 132-143.

Brasil. (2002). Doenças relacionadas com o trabalho: diagnóstico e condutas: manual de procedimentos para os serviços de saúde. Brasília, DF: Ministério da Saúde.

Codo, W., Soratto, L., \& Vasques-Menezes, I. (2004). Saúde mental e trabalho. In J. E. Zanelli, J. E. Borges-Andrade \& A. V. B. Bastos, Psicologia, organizações e trabalho no Brasil (pp. 276-299). Porto Alegre: Artmed.

Couto, H. de A., \& Moraes, L. F. R. de. (2000). Limites do homem. Revista Proteção, 40-44.

Doef, M. V. Der, \& Maes, S. (1999). The job demand-control (-support) model and psychological well-being: A review of 20 years of empirical research. Work \& Stress, 13(2), 87-114.

El-Batawi, M. A. (1988). Problemas de salud psicosociales de los trabajadores en los países en desarrollo. In R. Kalimo, M. A. El-Batawi \& C. L. Cooper, Los factores psicosociales en el trabajo y su relación con la salud (pp. 15-20). Genebra: OMS.

Fernandes, S. R. P. (1999). Transformações no mundo do trabalho e a saúde psíquica: a ótica do estresse ocupacional. Organizações \& Sociedade, 6(16), 67-75.

Figueiras, J. C., \& Hippert, M. I. (2002). Estresse: possibilidades e limites. In M. das G. Jacques \& W. Codo (Orgs.), Saúde mental e trabalho: leituras (pp. 12-129). Petrópolis, RJ: Vozes.

Folkman, S., \& Lazarus, R. S. (1980). An analysis of coping in a middle aged community sample. Journal of Health and Social Behavior, 21, 219-239.

Kalimo, R. (1988). Los factores psicosociales y la salud de los trabajadores: panorama general. In R. Kalimo, M. El-Batawi \& C. L. Cooper, Los factores psicosociales en el trabajo y su relación con la salud (pp. 3-8). Genebra: OMS.

Karasek Jr., R. A. (1979). Job demands, job decision latitude, and mental strain: Implications for job redesign. Administrative Science Quarterly, 24 285-308.

Karasek Jr., R. A., \& Theorell, T. (1990). Healthy work: stress productivity and the reconstruction of working life. New York: Basic Books.

Karlquist, L., Hagberg, M., Koster, M., Wenemark, M., \& Nell, R. (2003). Excessive physical demands in modern work life and characteristics of work and living conditions of persons at risk. Scandinavian Journal of Work, Environment \& Health, 29(5), 363-377.

Levi, L. (1988). Definiciones y aspectos teóricos de la salud en relación con el trabajo. In R. Kalimo, M. El-Batawi \& C. L. Cooper, Los factores psicosociales en el trabajo y su relación con la salud (pp. 9-14). Genebra: OMS.

Levi, L. (1998). Stress in organizations: Theoretical and empirical approaches. In C. L. Cooper (Ed.), Theories of organizational stress (pp. 129-174). New York: Oxford University Press.

Lima, M. E. A. (2002). Esboço de uma crítica à especulação no campo da saúde mental e trabalho. In M. das G. Jacques \& W. Codo, Saúde mental \& trabalho: leituras (pp. 50-81). Petrópolis, RJ: Vozes.

Marmot, M. (1999). Importance of the psychosocial environment in epidemiological studies.Scandinavian Journal of Work, Environment \& Health, 25(4), 49-53.

Martinez, S. V. (2004). Riesgo psicosocial: el modelo demanda control - apoyo social. Recuperado em 1 de julho de 2004, da http://www.mtas.es/msm/mp/603.htm

Monteiro, M. S., \& Gomes, J. da R. (1998). Reestruturação produtiva e saúde do trabalhador: um estudo de caso. Cadernos Saúde Pública, 14(2), 345-353.

National Institute for Occupational Safety and Health. (2004). Stress at work. Recuperado em 26 de julho de 2004, da http:// stress.cdc.gov/niosh/stresswk.html

Piko, B. F. (2003). Psychosocial work environment and psychosomatic health of nurses in Hungary. Work \& Stress, 17(1), 93-100.

Rodríguez, I., Bravo, M. J., Peirò, J. M., \& Schoufeli, W. (2001). The demands - control - support model, locus of control and job dissatisfaction: A longitudinal study. Work \& Stress, 15(2), 97-114.

Seligmann-Silva, E. (1997). Saúde mental e automação: a propósito de um estudo de caso no setor ferroviário. Cadernos Saúde Pública, 13(2), 95-109.

Siegrist, J. (1998). Adverse health effects of effort: Reward imbalance at work. In C. L. Cooper (Ed.), Theories of organizational stress (pp. 190-204). New York: Oxford University Press.

Theorell, T. (1998). Job characteristics in a theoretical and practical health context. In C. Cooper (Ed.), Theories of organizational stress (pp. 205-219). New York: Oxford University Press.

Tsutsumi, A., Kayaba, K., Theorell, T., \& Siegrist, J. (2001). Association between job and depression among japanese employees threatened by job loss in a comparison between two complementary. Scandinavian Journal of Work, Environment \& Health, 27(2), 146-153.

Villalobos, J. O. (2004). Estrés y trabajo. Instituto Mexicano del Seguro Social. Recuperado em 21 de janeiro de 2004, da Medspain (Revista de Medicina y Salud): www.medspain. com/n3-feb99/stress.htm 